\title{
Eisenia fetida Protease-III-1 Functions in Both Fibrinolysis and Fibrogenesis
}

\author{
Jing Zhao, ${ }^{1,2}$ Rong Pan, ${ }^{1,2}$ Jian He, ${ }^{1,2}$ Ying Liu, ${ }^{1,2}$ Dong-Feng Li, ${ }^{3}$ and Rong-Qiao He ${ }^{1,2}$ \\ ${ }^{1}$ State Key Laboratory of Brain and Cognitive Science, Institute of Biophysics, Baiao Pharmaceuticals Beijing C.L., \\ Chinese Academy of Sciences, 15 Da Tun Road, Chao Yang District, Beijing 100101, China \\ ${ }^{2}$ Graduate School, Chinese Academy of Sciences, Beijing 100039, China \\ ${ }^{3}$ College of Life Science, South China Normal University, Guangzhou 510631, China
}

Received 10 December 2006; Accepted 19 March 2007

Recommended by Abdelali Haoudi

The fibrinolytic function of earthworm protease-III-1 (EfP-III-1) has been studied in recent years. Here, we found that Ef P-III-1 acted not only in fibrinogenolysis, but also in fibrogenesis. We have used Ef P-III-1 to hydrolyze fibrinogen, and to activate plasminogen and prothrombin. Based on the N-terminal sequences of the hydrolytic fragments, Ef P-III- 1 was showed to specifically recognize the carboxylic sites of arginine and lysine. Analyses by fibrinogenolysis mapping and amino acid sequencing revealed that the isozyme could cleave the alpha, beta, and gamma chains of fibrinogen, showing a high $\alpha$-fibrinogenase, moderate $\beta$ fibrinogenase, and low $\gamma$-fibrinogenase activities. Interestingly, Ef P-III-1 activated plasminogen and released active plasmin, suggesting a tPA-like function. Furthermore, Ef P-III-1 showed a factor Xa-like function on prothrombin, producing alpha-thrombin. The function in both activating prothrombin and catalyzing fibrinogenolysis suggests that EfP-III-1 may play a role in the balance between procoagulation and anticoagulation.

Copyright (c) 2007 Jing Zhao et al. This is an open access article distributed under the Creative Commons Attribution License, which permits unrestricted use, distribution, and reproduction in any medium, provided the original work is properly cited.

\section{INTRODUCTION}

Earthworms have been made as a drug (usually in dried powder for oral administration) to improve blood circulation and to treat apoplectic stroke for tens of centuries. Early in 1878, Frédéricq found the alimentary tract of earthworm excreting a type of protease [1]. As described by Charles Darwin in 1883, earthworm digestive fluids can dissolve fibrin (see "The Formation of Vegetable Mould through the Action of Worms"). In the 1980s, groups of fibrinolytic isozymes were isolated from different earthworm species [2-4], such as Eisenia fetida proteases (EfP) $[5,6]$ and Lumbricus rubellus proteases $(\operatorname{LrP})[1,7]$. The isozymes of Eisenia fetida have been purified and made into a drug in capsule to treat clotting diseases. This preparation is stable and has high fibrinolytic activity with a little side effect in hemorrhage [810]. Similar to LrP-III-1 [11, 12], Ef P-III-1 has the highest fibrinolytic activity among the isozymes and has high stability [13]. Moreover, Ef P-III-1 acts as a tissue plasminogen activator (tPA)-like activator and initiates the plasminantithrombus pathway [14-16]. Therefore, the structural and functional characteristics of EfP-III-1 have become interesting and significant $[10,12,17-20]$.
Recently, the crystal structure of EfP-III-1 (EFEb) at a resolution of $2.06^{\circ} \mathrm{A}$ has been solved, and the structural analysis shows that Ef P-III-1 should be classified as a trypsin from earthworm [10]. However, the structure of this isozyme is different from other trypsins. As a trypsin-like protease, Ef P-III-1 contains two chains: an N-pyroglutamated light chain and an $\mathrm{N}$-glycosylated heavy chain. The heavy chain contains a novel structural motif, an eight-membered ring resulting from a disulfide bridge between two neighboring cysteine residues, and a cis-peptide bond exists between these two cysteine residues. The crystal structure of EfP-III-1 provides the structural basis for its high level of stability and reveals its complicated posttranslational modifications in the earthworm.

In this laboratory, eight trypsin-like isozymes with fibrinolytic activity were isolated from Eisenia fetida through a stepwise-purified procedure: sulfate ammonia precipitation, affinity chromatography with a Sepharose-4B column coupled with soybean trypsin inhibitor (SBTI), and ionic chromatography with a DEAE-cellulose-52 column [21]. Interestingly, all the proteases were glycosylated. In the assay with the substrates of trypsin, chymotrypsin, and elastase 
[17], Ef P-III-1 was demonstrated as a trypsin-like protease. So far, however, no detailed maps have been provided for the protease involved in fibrinogenolysis and plasminogen activation. Furthermore, the effect of EfP-III-1 on procoagulation has not been investigated yet. This work is concerned with the functions of Ef P-III-1 in both fibrinolysis and fibrogenesis.

\section{MATERIALS AND METHODS}

\subsection{Purification and assay of EfP-III-1}

Ten mg of crude earthworm proteases, prepared as described [17], was obtained from the ammonium sulfate precipitation of Eisenia fetida. EfP-III-1 was purified on a 4aminobenzamidine dihydrochloride-coupled Sepharose CL6B affinity column (Pharmacia/ Pfizer Canada) eluted with a gradient of denaturant (from 0.1 to $1 \mathrm{M}$ ) through a BioRad Gradient Maker [22]. The fractions were assayed by a chromogenic substrate (from Roche Switzerland) as described [23]. The active eluate was pooled and dialyzed against 0.01M Tris-HCl buffer ( $\mathrm{pH} 8.0$ ). Then the purified Ef P-III-1 was lyophilized and stored at $-20^{\circ} \mathrm{C}$ before use. To check the purity of the isozyme, the protein sample was resuspensed in $10 \mathrm{mM}$ Tris- $\mathrm{HCl}$ buffer ( $\mathrm{pH} 8.0$ ) and electrophoresed on $12 \%$ SDS-PAGEs, then the gels were stained with Coomassie blue and silver, respectively. The protein concentration was determined by the bicinchoninic acid (BCA) protein assay with bovine serum albumin as the standard (Pierce, Ill, USA).

The fibrinolytic activity was measured by the light scattering method as described by Zhou et al. [24]. The isozyme $(0.1 \mu \mathrm{M}$ final concentration) was incubated with fibrinogen $(0.25 \mathrm{mg} / \mathrm{mL}$ final concentration) in $0.05 \mathrm{M}$ Tris- $\mathrm{HCl}$ buffer $(\mathrm{pH} 7.4)$ at $25^{\circ} \mathrm{C}$ for 10 minutes. Then thrombin $(6 \mathrm{U} / \mathrm{mL}$, Sigma Co., Mo, USA) was added to the mixture, followed by measurement of the Rayleigh-light scattering at $480 \mathrm{~nm}$ on a fluorescence spectrophotometer (Hitachi F-4500). One enzymatic unit was defined as the amount of enzyme causing the conversion of $1 \mu \mathrm{M}$ of substrate per minute per mg of protein at $25^{\circ} \mathrm{C}$. The standard earthworm protease with a specific activity of $2.5 \times 10^{3} \mathrm{U}$ (Chinese National Pharmacopoeia) was purchased from the Institute for the National Control of Pharmacological and Biological Products, China.

\subsection{Hydrolysis of fibrinogen, plasminogen, and prothrombin by EfP-III-1}

EfP-III-1 $(0.1 \mu \mathrm{M}$ final concentration) was incubated with human fibrinogen, plasminogen, or prothrombin $(2 \mathrm{mg} / \mathrm{mL}$ final concentration, Sigma Co., Mo, USA) in $0.05 \mathrm{M}$ Tris$\mathrm{HCl}(\mathrm{pH} 8.3)$ at $25^{\circ} \mathrm{C}$. Aliquots were taken at different time intervals for SDS-PAGE, each of the bands was transferred onto a polyvinylidene difluoride membrane (Gelman, USA) and then each band was cut off from the membrane for the amino acid sequencing [25]. On the basis of the N-terminal sequence of each digested fragment, the cleavage sites were obtained by reference against the complete amino acid sequences of human fibrinogen [26], prothrombin [27], and bovine plasminogen [28]. The immobilized enzyme, prepared as described $[20,29]$, was also used for the hydrolysis. Fifty microliters of Sepharose CL-6B coupled with EfP-III-1 was put in an eppendorf tube $(0.5 \mathrm{~mL})$. Fibrinogen $(1 \mathrm{mg} / \mathrm{mL}$ final concentration) was dissolved in Tris- $\mathrm{HCl}$ buffer $(\mathrm{pH}$ 8.3 ), added to the eppendorf tube (total volume $200 \mu \mathrm{L}$ ), and placed on a shaker at $25^{\circ} \mathrm{C}$. Aliquots were taken by spinning down the reaction mixture at different time intervals, and the supernatants $(20 \mu \mathrm{L})$ were subjected to electrophoresis and amino acid sequencing.

\subsection{Assays of EfP-III-1, plasmin, and thrombin}

The activities of Ef P-III-1, plasminogen, and prothrombin were measured by the light scattering intensity on a fluorescence spectrophotometer (Hitachi F-4500). (I) Fibrogenesis: fibrinogen $(2.5 \mathrm{mg} / \mathrm{mL}$ final concentration) was incubated in $0.05 \mathrm{M}$ Tris- $\mathrm{HCl}$ buffer $(\mathrm{pH} 7.4)$ at $25^{\circ} \mathrm{C}$ for 10 minutes before addition of thrombin $(0.2 \mathrm{U})$, followed by the measurement of the Rayleigh-light scattering at 480nm [24]. (II) Assay of EfP-III-1: conditions were as for (I), except that EfPIII- $1(0.1 \mu \mathrm{M}$ as final concentration $)$ and thrombin were both added to fibrinogen. (III) Activation of plasminongen: conditions were referred to (II), except that plasminogen (3U) was incubated with EfP-III- 1 at $25^{\circ} \mathrm{C}$ for 10 minutes, and then added to fibrinogen in the presence of thrombin. (IV) Activation of prothrombin: instead of thrombin, prothrombin $(1 \mathrm{mg} / \mathrm{mL})$ was incubated with EfP-III- 1 at $25^{\circ} \mathrm{C}$ for 10 minutes and added into fibrinogen under the same conditions. Prothrombin without the incubation was used as control.

\subsection{Transmission electron microscopy}

Ef P-III-1 $(0.1 \mu \mathrm{M}$ final concentration) was incubated with fibrinogen or prothrombin $(2 \mathrm{mg} / \mathrm{mL}$ final concentration) for 30 minutes at $25^{\circ} \mathrm{C}$. Aliquots $(10 \mu \mathrm{L})$ were observed by electron microscopy. Samples were adsorbed (0.25-15 minutes) onto 300-mesh Formvar /carbon-coated copper grids. The resultant grids were washed with water, stained with $2 \%$ uranyl acetate (1 minute), washed again with water, blotted dry, and viewed in a JEOL JSM-5600 electron microscope operated at $65 \mathrm{kV}$ (Japan Electron Optics Laboratory Co., Ltd., Japan). Random images from each sample were captured onlm at 5000- to 50000-fold magnication, digitized, calibrated, and imported into Optimas 6.5.1 for quantitation of filament length and number as described previously [30].

\section{RESULTS}

\subsection{Purification and assay of EfP-III-1}

Ef P-III-1 was purified by the affinity chromatography and it showed a single protein band on the gel with an apparent molecular mass of $\sim 34 \mathrm{kd}$ after purification (Figure 1). Light scattering assay showed that the specific activity of the purified enzyme was $33 \times 10^{4} \mathrm{U}$. Sequencing of the 


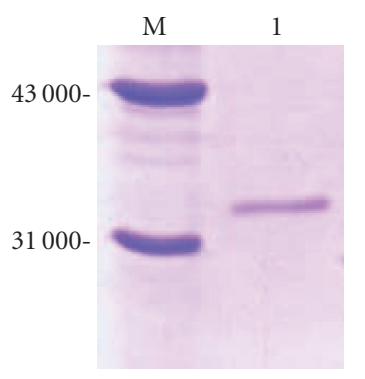

(a)

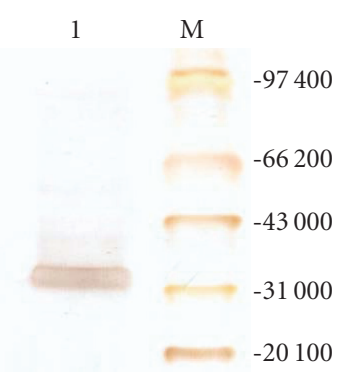

(b)
Figure 1: Purification of EfP-III-1. EfP-III-1 ( 34kd, lane 1) after purification was electrophoresed on a $12 \%$ SDS-PAGE. Then the gel was stained with Coomassie blue (a) or silver (b). M represents the low-molecular-mass protein markers.

$\mathrm{N}$-terminal region of $E f$ P-III-1 revealed amino acid sequence identical to LrP-III-1 (IVGGIEAR...) [12]. The unique sequence also demonstrated the high purity of EfP-III-1.

\subsection{Hydrolysis of fibrin and fibrinogen}

In order to investigate the fibrinolytic activity of EfP-III1 , we used transmission electron microscopy to observe the morphological changes when fibrin was incubated with the protease. Fibrogenesis was clearly detected when thrombin was incubated with fibrinogen as shown in Figure 2(c). No observable fibrils could be detected after the incubation of fibrin with Ef P-III-1 (Figure 2(a)). Ef P-III-1 alone as a control also exhibited no fibrils under the electronic microscope (Figure 2(b)). This observation suggests that Ef P-III-1 has a high fibrinolytic activity.

Furthermore, we hydrolyzed fibrinogen in the presence of Ef P-III-1 to reveal the map of the hydrolysis in which six major distinct fragments with different apparent molecular masses $(42,31,28,25,21$, and 13kd, resp.) have been found (Figures 2(d), 2(e)). On the SDS-PAGE, F-b appeared at the initial stage of digestion, followed by the appearance of Fd, F-e, F-a, F-f, and F-g whose band densities in the gel increased with time, and no further degradation could be detected when the reaction was prolonged to over 120 minutes (Figures 2(d), 2(e)). This observation suggests that these five products were stable under the assay conditions. Moreover, another stable band called F-c contains at least two peptides. As exhibited in Table 1, F-d, F-e, and F-f were hydrolytic products from the $\alpha$-chain of fibrinogen with cleavage sites at $\mathrm{R}_{252}-\mathrm{G}_{253}, \mathrm{R}_{19}-\mathrm{V}_{20}$, and $\mathrm{K}_{429}-\mathrm{V}_{430}$, respectively; F-a and Fb came from $\gamma$-chain and contained $\sim 410$ and $\sim 300$ amino acid residues, respectively. It should be noted that $\alpha$-chain was cleaved so rapidly (lane 3, Figure 2(d)) that the band disappeared within 5 minutes under such conditions. Consequently, hydrolysis of $\alpha$-chain was further carried out using immobilized Ef P-III-1 (Figure 2(f)). The density of $\alpha$-chain band in the gel gradually decreased during the hydrolysis. As shown in Figure 2(g), four distinct fragments (F-a, F-b', Fc', and F-d') were released, with different apparent molecular
TABLE 1: Cleavage sites on fibrinogen, plasminogen, and prothrombin by EfP-III-1.

\begin{tabular}{|c|c|c|c|}
\hline \multirow{2}{*}{ Fragment } & \multicolumn{3}{|c|}{ EfP-III-1 } \\
\hline & Fibrinogen* & Plasminogen** & Prothrombin*** \\
\hline $\mathrm{a}$ & $\gamma-Y_{1}$ VATR & $\mathrm{D}_{1} \mathrm{LLDD}$ & $\mathrm{PR}_{3} \downarrow \mathrm{ANTF}$ \\
\hline $\mathrm{b}$ & $\gamma-Y_{1}$ VATR & $\mathrm{GR}_{557} \downarrow$ IVGG & $\mathrm{PR}_{158} \downarrow \mathrm{SEGS}$ \\
\hline c & - & - & - \\
\hline d & $\alpha-\mathrm{TR}_{252} \downarrow \mathrm{GGST}$ & - & - \\
\hline e & $\alpha-\mathrm{PR}_{19} \downarrow$ VVER & - & $\mathrm{PR}_{158} \downarrow \mathrm{SEGS}$ \\
\hline $\mathrm{f}$ & $\alpha-\mathrm{EK}_{429} \downarrow$ VTSG & - & $\mathrm{GR}_{274} \downarrow$ TATS \\
\hline g & - & - & $\mathrm{ER}_{396} \downarrow$ NIEK \\
\hline $\mathrm{h}$ & - & - & $\mathrm{PR}_{287} \downarrow \mathrm{TFGS}$ \\
\hline $\mathrm{i}$ & 一 & - & $\mathrm{PR}_{3} \downarrow \mathrm{ANTF}$ \\
\hline
\end{tabular}

$*$, **, and $* * *$ indicate that the amino acid sequences are referred to in [25-27], respectively.

masses $(33,31,28$, and $22 \mathrm{kd})$. With the same N-terminus as the intact $\alpha$-chain [26], F-a' was initially released in 5 minutes during the reaction. The other three fragments had an identical cleavage site at $\mathrm{R}_{19}-\mathrm{V}_{20}$. According to the densities of the protein bands on the SDS-PAGE, hydrolysis of $\alpha$-chain was the fastest, and hydrolysis of $\beta$-chain was faster than that of $\gamma$-chain. This indicated that EfP-III-1 possesses strong $\alpha$-fibrinogenase, moderate $\beta$-fibrinogenase, and weak $\gamma$-fibrinogenase activities.

The light scattering assay was carried out to detect whether EfP-III-1 could cleave fibrinogen. Compared with the changes in the intensity of light scattering of the mixture containing fibrinogen and thrombin (curve 1, Figure 2(h)), the fibrin formation was clearly repressed when EfP-III-1 was added (curve 2, Figure 2(h)). To investigate which is the initial digestion target of the protease, fibrinogen or fibrin, we preincubated fibrinogen with EfP-III-1 for 10 minutes, and then added thrombin. As shown in Figure 2(h), the inhibition of fibrogenesis could be observed in the presence of Ef P-III-1, regardless of preincubation with fibrinogen or not. However, the relaxation time was remarkably prolonged ( $\sim 100$ seconds) when the protease was preincubated with fibrinogen (curve 3, Figure 2(h)). The inhibition of fibrin formation could be distinctly delayed, suggesting that EfPIII-1 preferably cleaved fibrinogen under such conditions.

\subsection{Activation of plasminogen}

To check the effect of EfP-III-1 on plasminogen, we used Ef P-III-1 to activate plasminogen and observed two resultant fragments $\mathrm{P}-\mathrm{a}$ and P-b (Figure 3, apparent molecular masses: $\sim 60$ and $\sim 30 \mathrm{kd}$, resp.). Cleavage at $\mathrm{R}_{557}-\mathrm{I}_{558}$ resulted in the release of P-b (Figures 3(a), 3(b)), whose first six amino acids were identical to those of the catalytic domain (or microplasminogen) of activated plasmin [28]. This cleavage site $\left(\mathrm{R}_{557^{-}}\right.$ $\mathrm{I}_{558}$ ) was also recognized by tPA (Figure 3(c)). 


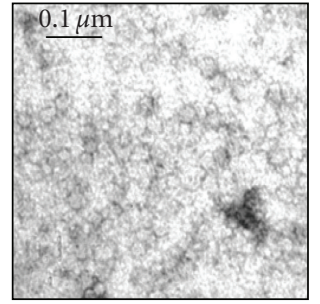

(a)

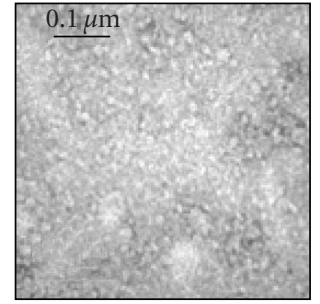

(b)

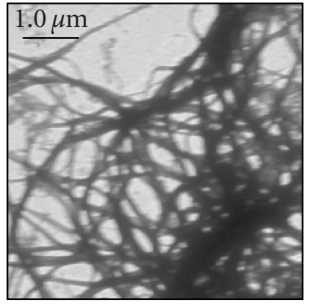

(c)

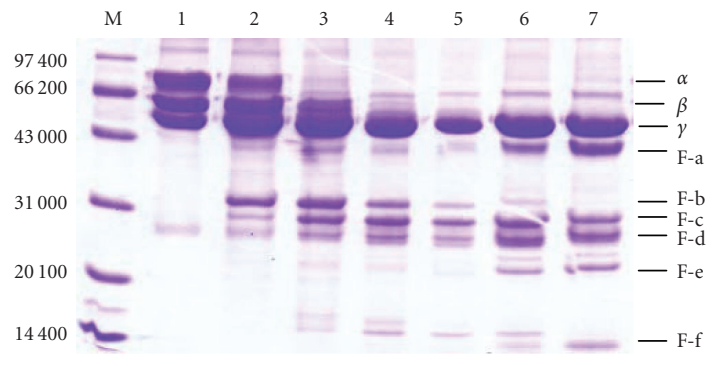

(d)

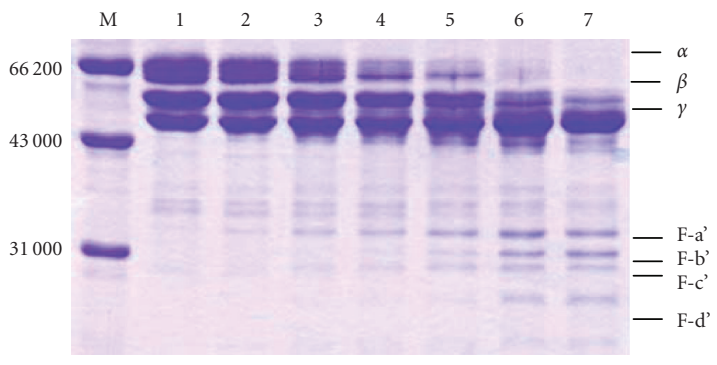

(f)

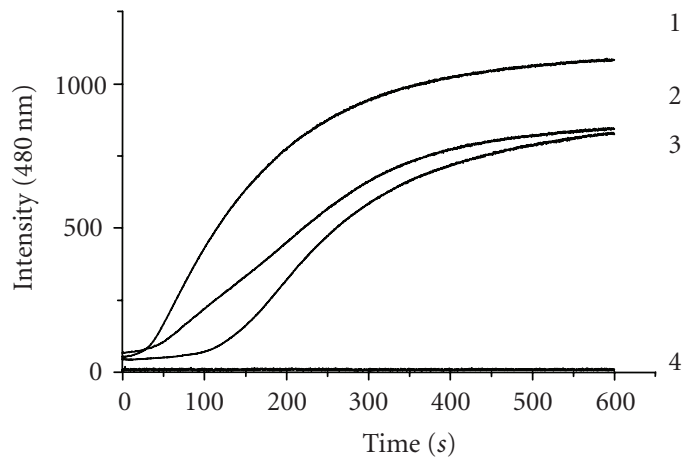

(h)

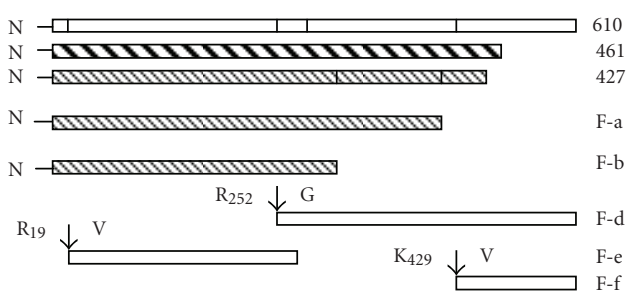

(e)

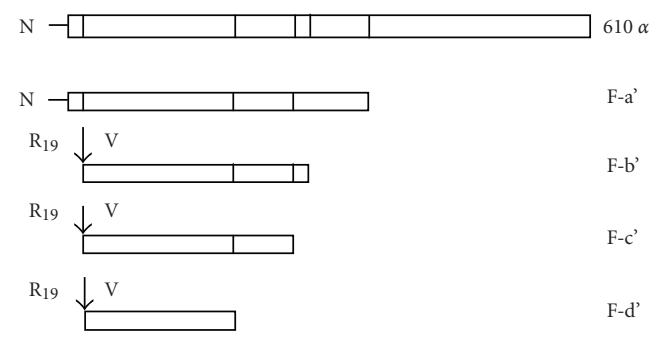

(g)

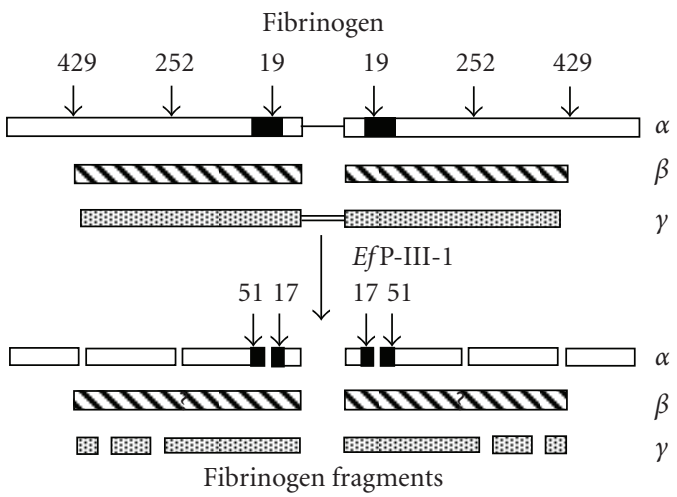

(i)

FIgure 2: Hydrolysis of fibrinogen by EfP-III-1. EfP-III-1 $(0.1 \mu \mathrm{M}$ final concentration) was incubated with human fibrinogen $(2 \mathrm{mg} / \mathrm{mL}$ in $50 \mathrm{mM}$ Tris- $\mathrm{HCl}$ buffer, $\mathrm{pH} 7.4)$ at $25^{\circ} \mathrm{C}$. (a-c) Fibrinolysis in the presence of EfP-III-1 (a) was detected by electron microscopy. Ef P-III-1 (b) and fibrin alone (c) were used as controls. (d,e) 12\% SDS-PAGE showing fibrinolysis in the presence of EfP-III-1 (d). M: low-molecularmass protein markers; lane 1: fibrinogen which consists of three polypeptide chains: $\alpha$ (66kd), $\beta$ (54kd), and $\gamma$ chain (48kd) as control; lanes 2 through 7: aliquots were taken at $0,5,15,30,60$, and 120 minutes during the hydrolysis. (e) The fibrinolytic fragments (F-a to F-f) in the presence of $E f$ P-III-1 (e). (f,g) fibrinolysis in the presence of immobilized Ef P-III-1 under the same conditions. $\alpha$-Fibrinogen fragments were indicated by F-a' to F-d' (g). Fibrinolytic activity $\left(2.5 \mathrm{mg} / \mathrm{mL}, 10\right.$ minutes, $25^{\circ} \mathrm{C}$ ) was measured by light scattering assay (h). Thrombin was added to fibrinogen in the absence of Ef P-III- 1 as a control (curve 1); both Ef P-III-1 and thrombin were added to fibrinogen at the same time (curve 2); Ef P-III-1 was incubated with fibrinogen at $25^{\circ} \mathrm{C}$ for 10 minutes before thrombin was added (curve 3 ), and Tris- $\mathrm{HCl}$ buffer as a negative control (curve 4). Ef P-III- 1 cuts at $\mathrm{R}_{19}-\mathrm{V}_{20}$ and destroys the N-terminal 35-residue stretch (black) which is the key recognition motif for thrombin (i), according to C. Fuss et al. [31]. 
TABLE 2: Activities of fibrinogen, plasminogen, and prothrombin.

\begin{tabular}{lcc}
\hline Samples & $\begin{array}{c}\text { Relative activity } \\
(\%, \text { mean } \pm \text { SD })^{*}\end{array}$ & $\begin{array}{c}\text { Fibrin formation } \\
(\%, \text { mean } \pm \text { SD })^{* *}\end{array}$ \\
\hline $\begin{array}{l}\text { Fibrinogen }+ \\
\text { thrombin } \\
\begin{array}{l}\text { Fibrinogen }+ \\
\text { thrombin }+\end{array}\end{array}$ & $100 \pm 29.9$ \\
$\begin{array}{l}\text { EfP-III-1 } \\
\text { Fibrinogen }+ \\
\text { thrombin }+ \\
\text { plasminogen }\end{array}$ & $38.6 \pm 1.8$ & $77.8 \pm 3.8$ \\
$\begin{array}{l}\text { Fibrinogen }+ \\
\text { thrombin }+ \\
\begin{array}{l}\text { EfP-III-1 }+ \\
\text { plasminogen }\end{array}\end{array}$ & $81.1 \pm 79.9$ & $69.9 \pm 3.6$ \\
& $27 \pm 9.9$ & $5.3 \pm 2.4$ \\
$\begin{array}{l}\text { EfP-III-1 }+ \\
\text { prothrombin }+ \\
\text { fibrinogen } \\
\text { Prothrombin }+ \\
\text { fibrinogen }\end{array}$ & $31.9 \pm 1.5$ & \\
\hline
\end{tabular}

* Relative activity was based on the max slope of the reaction curve. ** Fibrin formation was calculated with the intensity of the sample at 10 minutes.

*** Refer to Figure 3(d), line 2.

The activation of plasminogen by EfP-III-1 was assayed with the light scattering method (Figure $3(\mathrm{~d})$ ). As shown in Table 2, the relative light scattering intensity of the reaction mixture (fibrinogen and thrombin) reached $77.8 \pm 3.8(\%)$ in 10 minutes when Ef P-III-1 was added. In particular, the light scattering intensity remarkably decreased $(5.3 \pm 2.4 \%)$ in the presence of both EfP-III-1 and plasminogen, though the intensity increased initially. Although the fibrin formation reached $69.9 \pm 3.6(\%)$ when plasminogen was present alone, the relative fibrogenesis activity $(81.1 \pm 7.9 \%)$ did not markedly decrease under the same conditions. This suggested that Ef P-III-1 activated plasminogen and released active plasmin.

\subsection{Activation of prothrombin}

So far, we have obtained evidence demonstrating the effect of the earthworm protease on fibrinolysis and activation of plasminogen. We further wondered whether Ef P-III1 was involved in procoagulation pathway. By electron microscopy, it is obviously exhibited that some fibrin deposits were formed from fibrinogen in the presence of prothrombin and EfP-III-1 (Figures 4(a), 4(b)). The result of electrophoresis showed that eight major hydrolytic fragments were released after prothrombin was incubated with EfP-III1 (Figures 4(d), 4(e)). As shown in Figure 4(c), fibrin formation was increased in the presence of both prothrombin and EfP-III-1. However, fibrin was hardly produced when prothrombin alone was added to fibrinogen (curve 4,
Figure 4(c)). This suggested that active thrombin was released during the incubation of prothrombin with Ef P-III-1.

\section{DISCUSSION}

The $\alpha$-chain of fibrinogen plays an important role in fibrin formation during the activation of fibrinogen to fibrin by thrombin (Figure 2(i)). The 35-residue stretch $\left(\mathrm{G}_{17}\right.$ to $\mathrm{M}_{51}$ ) in the $\mathrm{N}$-terminal region is a key recognition motif for thrombin [32]. Meanwhile, this stretch is essential for fibrin monomers to associate with each other, and to produce fibrin fibrils. We employed the immobilization of EfP-III-1 to hydrolyze the $\alpha$-chain of fibrinogen (Figure 2(f), 2(g)). Use of immobilized enzyme is based on the following reasons. (1) Hydrolysis of $\alpha$-chain was a rapid procedure resulting in a release of transient fragments, which could hardly be detected. (2) The immobilization of protease allows us to exclude the effects due to residual activity of Ef P-III-1. (3) After immobilization, the activity of EfP-III- 1 decreased to $40 \%-50 \%$, and the enzyme molecule became more resistant to heat, acidic and basic conditions, and denaturants [29]. (4) More importantly, the immobilization of protease enables us to control the reaction easily and to obtain reproducible results. (5) For EfP-III-1, immobilization did not change its substrate specificity (data not shown). As a result, we found that the peptide bond of $\mathrm{R}_{19}-\mathrm{V}_{20}$ was a cleavage site on $\alpha$-chain, and the site was close to $\mathrm{R}_{16}-\mathrm{G}_{17}$ recognized by thrombin [33], it may destroy the recognition stretch and obstruct the polymerization between fibrins.

Besides $\mathrm{R}_{19}-\mathrm{V}_{20}$, the $\alpha$-chain of fibrinogen has been cut by EfP-III-1 at other sites. According to the N-terminal sequencing of F-d and F-f, the cleavage sites were located at $\mathrm{R}_{252}-\mathrm{G}$ and $\mathrm{K}_{429}-\mathrm{V}$. Moreover, the sites were presumably located around $\mathrm{R}_{334}-\mathrm{P}_{335}$ (F-a'), $\mathrm{R}_{289}-\mathrm{N}_{290}$ (F-b'), $\mathrm{R}_{252}-\mathrm{G}_{253}$ (F$\left.c^{\prime}\right)$, and $\mathrm{R}_{199}-\mathrm{Q}_{200}$ (F-d') based on that EfP-III-1 specifically recognized the carboxylic sites of arginine and lysine which were demonstrated by the N-terminal sequencing of all the detected hydrolytic fragments (Table 1).

In addition, the $\mathrm{C}$-terminal region of the $\gamma$-chain of fibrinogen was also cleaved by the protease, producing F-a, F$b$, and some small undetectable peptides. Presumably, the hydrolytic sites in the $\mathrm{C}$-terminal region could be located around $\mathrm{R}_{419}-\mathrm{P}_{420}(\mathrm{~F}-\mathrm{a})$ and $\mathrm{K}_{302}-\mathrm{F}_{303}$ (F-b). Hydrolysis at these two predicted sites may also obstruct fibrin formation [34], due to the fact that the C-terminal region of fibrinogen is involved in several functional interactions, including fibrin polymerization [33]. Note that the enzyme digested fibrinogen at multiple hydrolytic sites, leading to a remarkable decrease in fibrinogen concentration. As described by Dempfle et al. [35] and Bovill et al. [36], the decrease of fibrinogen leads to an anticoagulation effect in circulation.

In our experiments, no fragment from $\beta$-chain could be detected in the presence of both native and immobilized EfPIII- 1 under the conditions. According to the amino acid sequence, $\beta$-fibrinogen is rich in arginine and lysine residues, which are probably vulnerable to EfP-III-1. Thus, it is likely that the $\beta$-chain of fibrinogen was hydrolyzed and the degraded bands disappeared within 15 minutes after addition 


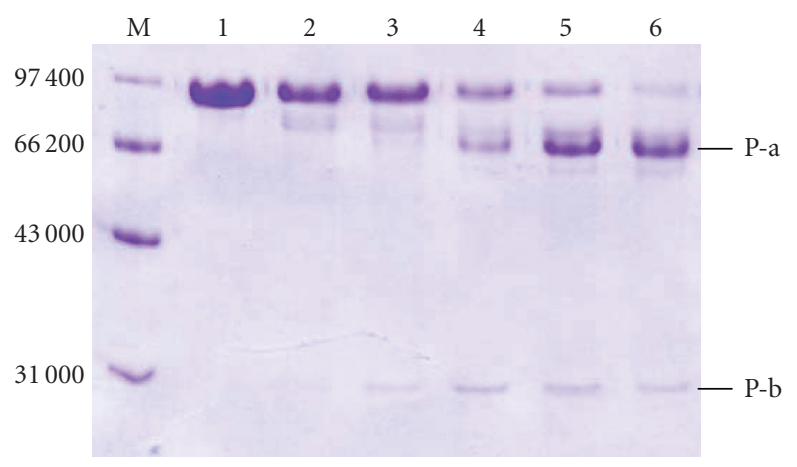

(a)

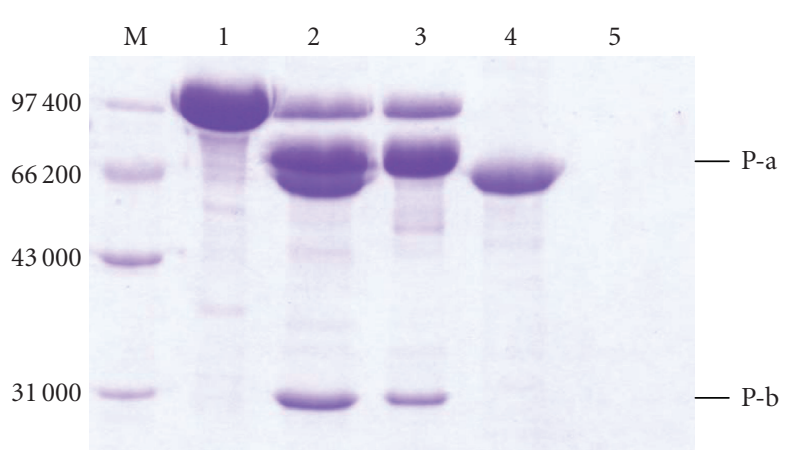

(c)

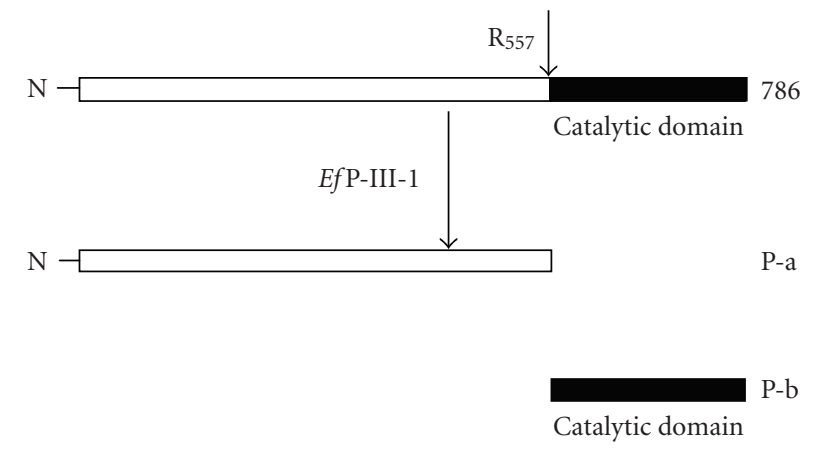

(b)

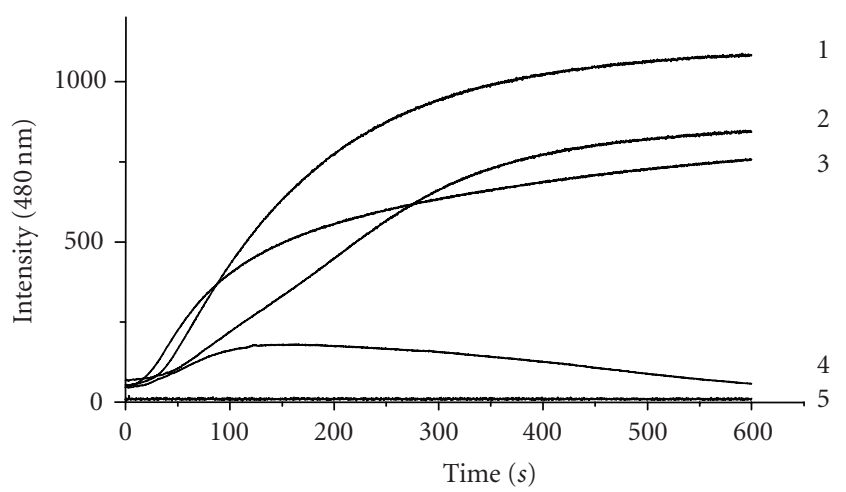

(d)

Figure 3: Activation of plasminogen by EfP-III-1. Plasminogen ( $2 \mathrm{mg} / \mathrm{mL}$ final concentration) dissolved in $50 \mathrm{mM}$ Tris-HCl buffer (pH 7.4) was incubated with Ef P-III- 1 at $25^{\circ} \mathrm{C}$. Aliquots were taken at different time intervals, and loaded on SDS-PAGE (12\%) (a). M shows low-molecular-mass protein markers; lane 1: plasminogen as control; lanes 2 through 6 represented the hydrolyzed fragments after 2, 5, 15, 30 , and 60 minutes. Cleavage of plasminogen after one-hour incubation with EfP-III- 1 at $25^{\circ} \mathrm{C}(\mathrm{b})$ represented the digested fragments P-a and P-b (catalytic domain or micropalsminogen). Plasminogen in the presence of tPA $(0.1 \mu \mathrm{M}$ final concentration) was used as a control (c). Lanes 1 through 3: plasminogen alone, with tPA, or with EfP-III-1; lane 4: tPA alone, and lane 5: EfP-III-1 alone. Enzymatic activity was measured by light scattering assay (d). Thrombin was added to fibrinogen in the presence of EfP-III-1 (curve 4, with plasminogen; curve 2, without plasminogen), and in the absence of EfP-III-1 (curve 3 with plasminogen). Tris-HCl buffer (curve 5) was used as control.

of EfP-III-1. In addition, the protease efficiently cleaves the $\alpha$-, $\beta$-, and $\gamma$-chains of fibrinogen at multiple sites in both the $\mathrm{N}$-terminal and C-terminal regions. However, the hydrolyzation of $\alpha$-chain is faster than that of $\beta$-chain, and much faster than that of $\gamma$-chain under the same conditions (Figure 2). This indicates that $E f$ P-III- 1 acts as a strong $\alpha$-fibrinogenase, a moderate $\beta$-fibrinogenase, and a weak $\gamma$-fibrinogenase.

The fibrinolytic system contains a proenzyme plasminogen which is converted to active enzyme plasmin by the action of plasminogen activators. Plasminogen activation by tPA is the most important mechanism in removing fibrin excess [37]. tPA specifically recognizes $\mathrm{R}_{557}-\mathrm{I}_{558}$ on plasminogen and releases active plasmin. The cleavage site on plasminogen by EfP-III- 1 was the same as tPA (Figure 3(a), 3(b)) [28], and no other cleavage sites could be detected under our assay conditions. This indicates that EfP-III-1 is a tPA-like protease.

Eight major hydrolytic fragments were released after prothrombin was incubated with EfP-III-1 (Figures 4(d), 4(e)). The apparent molecular masses of the fragments (from T-a to $\mathrm{T}$-i) were approximately $70,52,48,46,37,33,29$, and $25 \mathrm{kd}$, respectively. The N-terminal sequences of the eight fragments indicated that $E f \mathrm{P}-\mathrm{III}-1$ recognized peptidyl bonds at $\mathrm{R}_{3}-\mathrm{A}_{4}$ (T-a, T-g), $\mathrm{R}_{158}-\mathrm{S}_{159}$ (T-e), $\mathrm{R}_{274}-\mathrm{T}_{275}$ (T-f), $\mathrm{R}_{396}-\mathrm{N}_{397}$ (T-g, detectable in both T-e and T-f), and $\mathrm{R}_{287}-\mathrm{T}_{288}(\mathrm{~T}-\mathrm{h})$. On the basis of the molecular masses of T-e $(\sim 35 \mathrm{kd})$ and T-i $(\sim 25 \mathrm{kda})$, we estimated that the cleavage sites at C-terminus were located around $\mathrm{R}_{493}-\mathrm{P}_{494}$ and $\mathrm{R}_{243}-\mathrm{N}_{244}$.

The coagulation cascade culminates in the conversion of prothrombin to active thrombin. This reaction is catalyzed by a multicomponent complex prothrombinase $[33,38]$. In the physiological environment, the activation of prothrombin by factor Xa generates the thrombin catalytic sites, and cleavage at $R_{274}$ and $R_{320}$ is required for thrombin formation $[27,39,40]$. In one pathway, human prothrombin is converted relatively slowly to $\alpha$-thrombin (274-287) in the presence of factor $\mathrm{Xa}$ and $\mathrm{Ca}^{2+}$ ions by the initial cleavage at residue $\mathrm{R}_{274}$ (consequently producing fragment 1.2 and prethrombin 2), followed by cleavage of prethrombin 2 at $\mathrm{R}_{320}$ (accordingly generating $\alpha$-thrombin). In our experiments, Ef P-III-1 degraded prothrombin at $\mathrm{R}_{274}$ in the absence of $\mathrm{Ca}^{2+}$, and released prethrombin 2 (T-f, Figure 4(e)). 


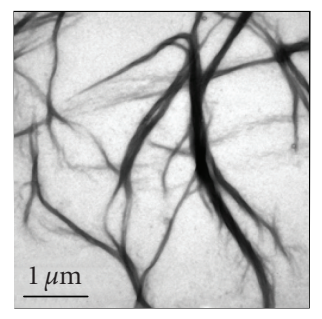

(a)

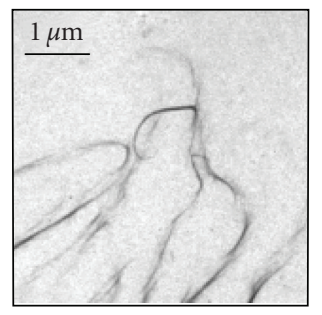

(b)

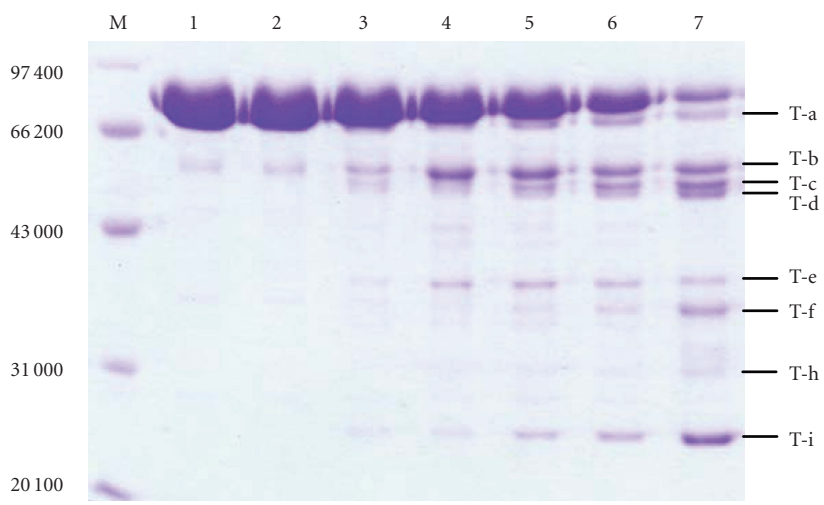

(d)

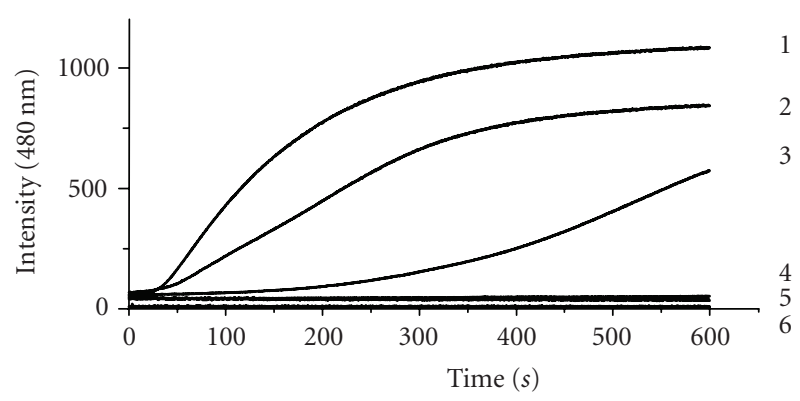

(c)

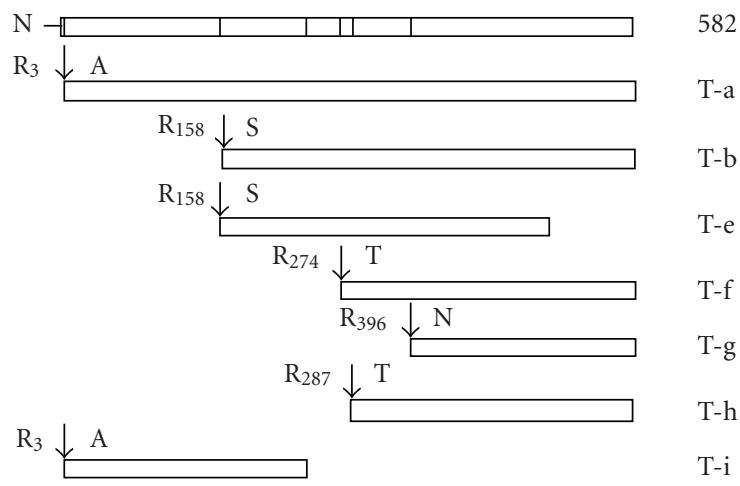

(e)

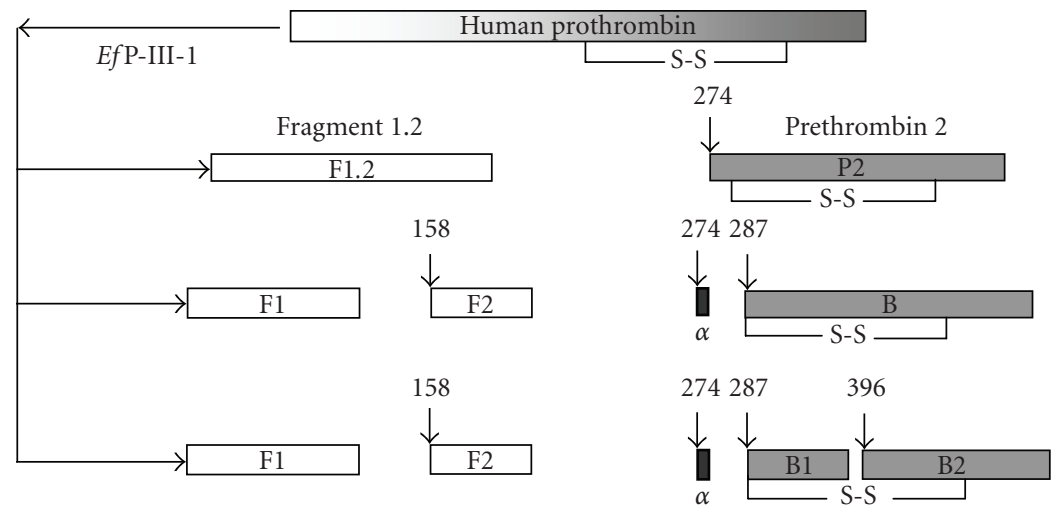

(f)

FIgure 4: Activation of prothrombin by EfP-III-1. Prothrombin $(2 \mathrm{mg} / \mathrm{mL}$ final concentration) in $50 \mathrm{mM}$ Tris- $\mathrm{HCl}$ buffer (pH 7.4$)$ was incubated with (a) or without (b) EfP-III-1 at $25^{\circ} \mathrm{C}$, and then added into fibrinogen and observed by electron microscopy. Activation of Ef P-III-1 on prothrombin was measured by light scattering assay (c). Thrombin was added to fibrinogen in the absence of Ef P-III-1 as a control (curve 1); prothrombin (curve 3) or thrombin (curve 2) was incubated with Ef P-III-1 before addition to fibrinogen; prothrombin (curve 4) or Ef P-III-1 (curve 5) was added to fibrinogen as controls, and Tris-HCl buffer as a negative control (curve 6). Prothrombin in the presence of Ef P-III-1 was electrophoresed on 12\% SDS-PAGE (d). M: low-molecular-mass protein markers; lane 1: prothrombin alone; lanes 2 through 7: hydrolyzed products after $0,5,15,30,60,120$ minutes, respectively. T-a to T-i represent the digested fragments of prothrombin in the presence of EfP-III-1 (e). Diagrammatic representation of prothrombin activation products produced by incubation with EfP-III-1 was shown in (f).

Cleavage at $\mathrm{R}_{287}$ produced $\alpha$-thrombin (Figure $4(\mathrm{f})$ ). Similar to the preference for residue $\mathrm{N}_{397}$ by thrombin, which produces the $\beta$-thrombin-like fragments [41], EfP-III-1 cleaves at residue $\mathrm{R}_{396}$. As shown in Figure $4(\mathrm{c})$, the activation of prothrombin by EfP-III-1 was time-dependent, and pro- thrombin was first degraded at $\mathrm{R}_{274}$ similar to the activation by factor Xa. Furthermore, cleavage at $\mathrm{R}_{287}$ on prethrombin 2 released active $\alpha$-thrombin for fibrogenesis [41]. Thus, EfPIII-1 had a prothrombinase-like function in activating prothrombin to produce $\alpha$-thrombin. 


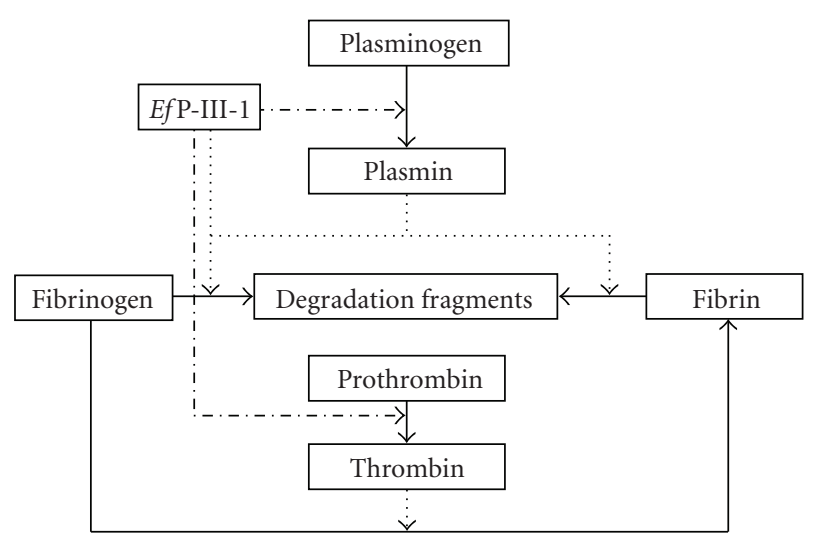

$\longrightarrow$ Give rise to

$-\rightarrow>$ Activation

….) Degradation

FIgURE 5: Roles of $E f$ P-III-1 in procoagulation and anticoagulation. Ef P-III-1 degrades fibrin(ogen) and activates not only plasminogen but also prothrombin. Activation is indicated by dashed lines, and degradation is indicated by dotted lines.

One problem we noted in our experiments was that Fc from fibrinogen was a mixture containing at least two peptides (Figure 2(d)), and so were T-c and T-d from prothrombin (Figure $4(\mathrm{~d})$ ). We have run SDS-PAGEs under both reducing and nonreducing conditions, but the mixed peptides could not be separated. There may be two reasons: (1) the molecular masses of the mixed peptides were similar, and/or (2) they were polymerized with each other and could not be separated with SDS, DTT, and $\beta$-mercaptoethanol.

Based on our data, the roles of Ef P-III-1 in procoagulation and anticoagulation can be summarized as follows (Figure 5): (1) to degrade fibrinogen and fibrin at both the $\mathrm{N}$-terminal and C-terminal regions (Figure 2); (2) to activate PLg and release active plasmin (Figure 3); and (3) to act in an Xa-like manner (Figure 4).

A clinical study of cerebral infarction has showed that earthworm proteases decreased some stroke scores in comparison with control groups [42]. According to clinical observations $[38,42-44]$, the activated partial thromboplastic time was prolonged, tPA activity and D-dimer levels increased, and the concentration of fibrinogen in blood decreased significantly. In recent years, the mixture of earthworm proteases including Ef P-III-1 has been made as an orally administered fibrinolytic agent to prevent and treat clotting diseases [11, 14, 45]. Compared with other drugs such as hirudin [46], tPA [47] and UK [48], "Lumbrokinase" (containing EfP-III-1) has relatively low side effects such as hemorrhage complications ( 2\%) [42]. Ef P-III-1, involving both fibrinolysis and fibrogensis, may play a role in balancing coagulation and anticoagulation in circulation. This may be one explanation for the observed low incidence of hemorrhage complications in clinical applications of earthworm proteases.

\section{ACKNOWLEDGMENTS}

We are grateful to Dr. Cen Wu, Dr. Qian Hua, and Ms. YaQun Zhang for their kind help and useful discussion. We thank Ms. Wei-Qun Shen and Mr. Bai-He Hu of Peking University for their excellent technical assistance. This work was supported by the National Natural Science Foundation of China (Grants 90206041 and 30170297), Major State Basic Research Development Program of China (973-Program, 2006CB500703), the Knowledge Innovation Project of the Chinese Academy of Sciences (KSCX2-SW214-1), the Chinese Academy of Sciences KC Wong Postdoctoral Research Award Fund. Jing Zhao and Rong Pan contributed equally to the paper.

\section{REFERENCES}

[1] H. Mihara, H. Sumi, H. Mizumoto, T. Yoneta, R. Ikeda, and M. Maruyama, "Oral administration of earthworm powder as a possiblethrombolytic therapy," in Recent Advances in Thrombosis and Fibrinolysis, K. Tanaka, Ed., pp. 287-298, Academic Press, New York, NY, USA, 1990.

[2] N. Nakajima, K. Ishihara, M. Sugimoto, H. Sumi, K. Mikuni, and H. Hamada, "Chemical modification of earthworm fibrinolytic enzyme with human serum albumin fragment and characterization of the protease as a therapeutic enzyme," Bioscience, Biotechnology and Biochemistry, vol. 60, no. 2, pp. 293300, 1996.

[3] J. Zhao, S. P. Qi, L. Li, J. Wu, and R.-Q. He, Earthworm Fibrinolytic Enzymes, vol. 30 of Studies in Natural Products Chemistry, Elsevier, North-Holland, The Netherlands, 2004.

[4] N. Nakajima, M. Sugimoto, and K. Ishihara, "Earthwormserine protease: characterization, molecular cloning, and application of the catalytic functions," Journal of Molecular Catalysis B: Enzymatic, vol. 23, no. 2-6, pp. 191-212, 2003.

[5] C. Wu and R. Fan, "A rapid and effective thrombolytic agent: e-PA," Acta Biophysica, vol. 2, p. 87, 1986.

[6] F. Wang, C. Wang, M. Li, L. Gui, J. Zhang, and W. Chang, "Purification, characterization and crystallization of a group of earthworm fibrinolytic enzymes from Eisenia fetida," Biotechnology Letters, vol. 25, no. 13, pp. 1105-1109, 2003.

[7] I. H. Cho, E. S. Choi, H. G. Lim, and H. H. Lee, "Purification and characterization of six fibrinolytic serine-proteases from earthworm Lumbricus rubellus," Journal of Biochemistry and Molecular Biology, vol. 37, no. 2, pp. 199-205, 2004.

[8] T. Hraenjak, M. Popovic, T. Bozic, et al., "Fibrinolytic and anticoagulative activities from the earthworm, Eisenia fetida," Comparative Biochemistry and Physiology-Part B: Biochemistry and Molecular Biology, vol. 119, no. 4, pp. 825-832, 1998.

[9] Y.-H. Li, M. Zhang, J.-C. Wang, S. Zhang, J.-R. Liu, and Q. Zhang, "Effects of absorption enhancers on intestinal absorption of lumbrokinase," Acta Pharmaceutica Sinica, vol. 41, no. 10, pp. 939-944, 2006.

[10] F. Wang, C. Wang, M. Li, et al., "Crystal structure of earthworm fibrinolytic enzyme component B: a novel, glycosylated two-chained trypsin," Journal of Molecular Biology, vol. 348, no. 3, pp. 671-685, 2005.

[11] H. Sumi, N. Nakajima, and H. Mihara, "A very stable and potent fibrinolytic enzyme found in earthworm Lumbricus rubellus autolysate," Comparative Biochemistry and PhysiologyPart B: Biochemistry and Molecular Biology, vol. 106, no. 3, pp. 763-766, 1993. 
[12] N. Nakajima, H. Mihara, and H. Sumi, "Characterization of potent fibrinolytic enzymes in earthworm, Lumbricus rubellus," Bioscience, Biotechnology and Biochemistry, vol. 57, no. 10, pp. 1726-1730, 1993.

[13] Q. Fan, C. Wu, L. Li, et al., "Some features of intestinal absorption of intact fibrinolytic enzyme III-1 from Lumbricus rubellus," Biochimica et Biophysica Acta: General Subjects, vol. 1526, no. 3, pp. 286-292, 2001.

[14] Y. S. Kim, M. K. Pyo, K. M. Park, B. S. Hahn, K. Y. Yang, and H. S. Yun-Choi, "Dose dependency of earthworm powder on antithrombotic and fibrinolytic effects," Archives of Pharmacal Research, vol. 21, no. 4, pp. 374-377, 1998.

[15] N. Nakajima, M. Sugimoto, and K. Ishihara, "Stable earthworm serine proteases: application of the protease function and usefulness of the earthworm autolysate," Journal of Bioscience and Bioengineering, vol. 90, no. 2, pp. 174-179, 2000.

[16] J.-S. Yang and B.-G. Ru, "Purification and characterization of an SDS-activated fibrinolytic enzyme from Eisenia fetida," Comparative Biochemistry and Physiology-Part B: Biochemistry and Molecular Biology, vol. 118, no. 3, pp. 623-631, 1997.

[17] J. Zhao, R. Xiao, J. He, et al., "In situ localization and substrate specificity of earthworm protease-II and protease-III-1 from Eisenia fetida," International Journal of Biological Macromolecules, vol. 40, no. 2, pp. 67-75, 2007.

[18] C. Wu, L. Li, J. Zhao, Q. Fan, W.-X. Tian, and R.-Q. He, "Effect of $\alpha_{2} \mathrm{M}$ on earthworm fibrinolytic enzyme III-1 from Lumbricus rubellus," International Journal of Biological Macromolecules, vol. 31, no. 1-3, pp. 71-77, 2002.

[19] J. Zhou, C. Wu, C. Wu, R. Fan, and R.-Q. He, "Characterization of activity and conformation of earthworm fibrinolytic enzyme III-1," Journal of Biochemistry, Molecular Biology and Biophysics, vol. 2, no. 3, pp. 195-199, 1999.

[20] J. Zhao, L. Li, C. Wu, and R.-Q. He, "Hydrolysis of fibrinogen and plasminogen by immobilized earthworm fibrinolytic enzyme II from Eisenia fetida," International Journal of Biological Macromolecules, vol. 32, no. 3-5, pp. 165-171, 2003.

[21] J. X. Wu, X. Y. Zhao, R. Pan, and R.-Q. He, "Glycosylated trypsin-like proteases from earthworm Eisenia fetida," International Journal of Biological Macromolecules, vol. 40, no. 5, pp. 399-406, 2007.

[22] R.-Q. He and J. Zhao, "A newly affinity chromatography technique for isolating monomer earthworm fibrinolytic enzyme," 2005, Chinese National Patent: ZL02116747.8.

[23] J. Zhou, K. Fan, C. Wu, and R.-Q. He, "Assay of lumbrokinase with a chromophoric substrate," Protein and Peptide Letters, vol. 4, no. 6, pp. 409-414, 1997.

[24] J. Zhou, K. Jiang, R.-Q. He, and Y. M. Han, "Assays of thrombin, hirudin and lumbrokinase with light scattering in the solution of fibrinogen," Acta Biophysica Sinica, vol. 13, pp. 531$535,1997$.

[25] J. Sambrook, E. F. Fritsch, and T. Maniatis, Molecular Cloning: A Laboratory Manual, Cold Spring Harbor Laboratory Press, New York, NY, USA, 2nd edition, 1989.

[26] R. F. Doolittle, K. W. Watt, B. A. Cottrell, D. D. Strong, and M. Riley, "The amino acid sequence of the $\alpha$-chain of human fibrinogen," Nature, vol. 280, no. 5722, pp. 464-468, 1979.

[27] S. J. Friezner Degen, R. T. A. MacGillivray, and E. W. Davie, "Characterization of the complementary deoxyribonucleic acid and gene coding for human prothrombin," Biochemistry, vol. 22, no. 9, pp. 2087-2097, 1983.

[28] J. Schaller, P. W. Moser, G. A. K. Dannegger-Müller, S. J. Rösselet, U. Kämpfer, and E. E. Rickli, "Complete amino acid sequence of bovine plasminogen. Comparison with hu- man plasminogen," European Journal of Biochemistry, vol. 149, no. 2, pp. 267-278, 1985.

[29] X.-Q. Wu, C. Wu, and R.-Q. He, "Immobilized earthworm fibrinolytic enzyme III-1 with carbonyldiimidazole activatedagarose," Protein and Peptide Letters, vol. 9, no. 1, pp. 75-80, 2002.

[30] D. L. Spector, R. D. Goldman, and L. A. Leinwand, Cells: A Laboratory Manual, Cold Spring Harbor Laboratory Press, New York, NY, USA, 1998.

[31] C. Fuss, J. C. Palmaz, and E. A. Sprague, "Fibrinogen: structure, function, and surface interactions," Journal of Vascular and Interventional Radiology, vol. 12, no. 6, pp. 677-682, 2001.

[32] D. Collen, "On the regulation and control of fibrinolysis. Edward Kowalski memorial lecture," Thrombosis and Haemostasis, vol. 43, no. 2, pp. 77-89, 1980.

[33] G. Tsurupa and L. Medved, "Fibrinogen $\alpha \mathrm{C}$ domains contain cryptic plasminogen and tPA binding sites," Annals of the New York Academy of Sciences, vol. 936, pp. 328-330, 2001.

[34] E. T. Bach-Gansmo, S. Halvorsen, H. C. Godal, and O. H. Skjonsberg, "Degradation of the $\alpha$-chain of fibrin by human neutrophil elastase reduces the stimulating effect of fibrin on plasminogen activation," Thrombosis Research, vol. 75, no. 3, pp. 307-317, 1994.

[35] C.-E. Dempfle, S. Argiriou, K. Kucher, H. Muller-Peltzer, K. Rubsamen, and D. L. Heene, "Analysis of fibrin formation and proteolysis during intravenous administration of ancrod," Blood, vol. 96, no. 8, pp. 2793-2802, 2000.

[36] E. G. Bovill, R. P. Tracy, T. E. Hayes, R. J. Jenny, F. H. Bhushan, and K. G. Mann, "Evidence that meizothrombin is an intermediate product in the clotting of whole blood," Arteriosclerosis, Thrombosis, and Vascular Biology, vol. 15, no. 6, pp. 754-758, 1995.

[37] H. W. Wang and C. F. Li, "Effect of earthworm plasminogen activator on coagulation and fibrinolysis systems," Tianjin Medicine, vol. 19, pp. 73-75, 1991.

[38] P. B. Tracy, L. L. Eide, and K. G. Mann, "Human prothrombinase complex assembly and function on isolated peripheral blood cell populations," Journal of Biological Chemistry, vol. 260, no. 4, pp. 2119-2124, 1985.

[39] Q. Dong, J. Qiao, and L.F. Shi, "The efficacy and safety of lumbrokinase capsule in treatment of cerebral infaretion," Chinese New Drugs Journal, vol. 13, no. 3, pp. 257-260, 2004.

[40] L. R. Jing and G. Z. Xu, "Dynamics of fibrinolysis and hemostasis in ischemic stroke patients, and the effects of lumbrokinase on those dynamics," Chinses Journal of New Drugs and Clinical Remedies, vol. 18, no. 1, pp. 48-50, 1999.

[41] S. Butenas, C. van't Veer, and K. G. Mann, "“Normal” thrombin generation,” Blood, vol. 94, no. 7, pp. 2169-2178, 1999.

[42] Y. P. Sun and R. Fan, "Fibrinolysis assay of lumbrokinase by intestinal administration in vivo," Capital Medicine, vol. 5, p. 17, 1998.

[43] Y.-D. Park, J.-W. Kim, B.-G. Min, J.-W. Seo, and J.-M. Jeong, "Rapid purification and biochemical characteristics of lumbrokinase III from earthworm for use as a fibrinolytic agent," Biotechnology Letters, vol. 20, no. 2, pp. 169-172, 1998.

[44] C. P. Cannon, "Hirudin in acute myocardial infarction," Journal of Thrombosis and Thrombolysis, vol. 1, no. 3, pp. 259-267, 1995.

[45] R. M. Califf, S. Mantell, C. Westawski, et al., "Experience with the use of tPA in the treatment of acute myocardial infarction," Annals of Emergency Medicine, vol. 17, no. 11, pp. 1176-1189, 1988. 
[46] B. Wiman, "Primary structure of peptides released during activation of human plasminogen by urokinase," European Journal of Biochemistry, vol. 39, no. 1, pp. 1-9, 1973.

[47] C. Duboscq, V. Genoud, M. F. Parborell, and L. C. Kordich, "Impaired clot lysis by rt-PA catalyzed mini-plasminogen activation," Thrombosis Research, vol. 86, no. 6, pp. 505-513, 1997.

[48] E. G. Bovill, R. P. Tracy, and T. E. Hayes, "Evidence that meizothrombin is an intermediate product in the clotting of whole blood," Arteriosclerosis, Thrombosis, and Vascular Biology, vol. 15, no. 6, pp. 754-758, 1995. 

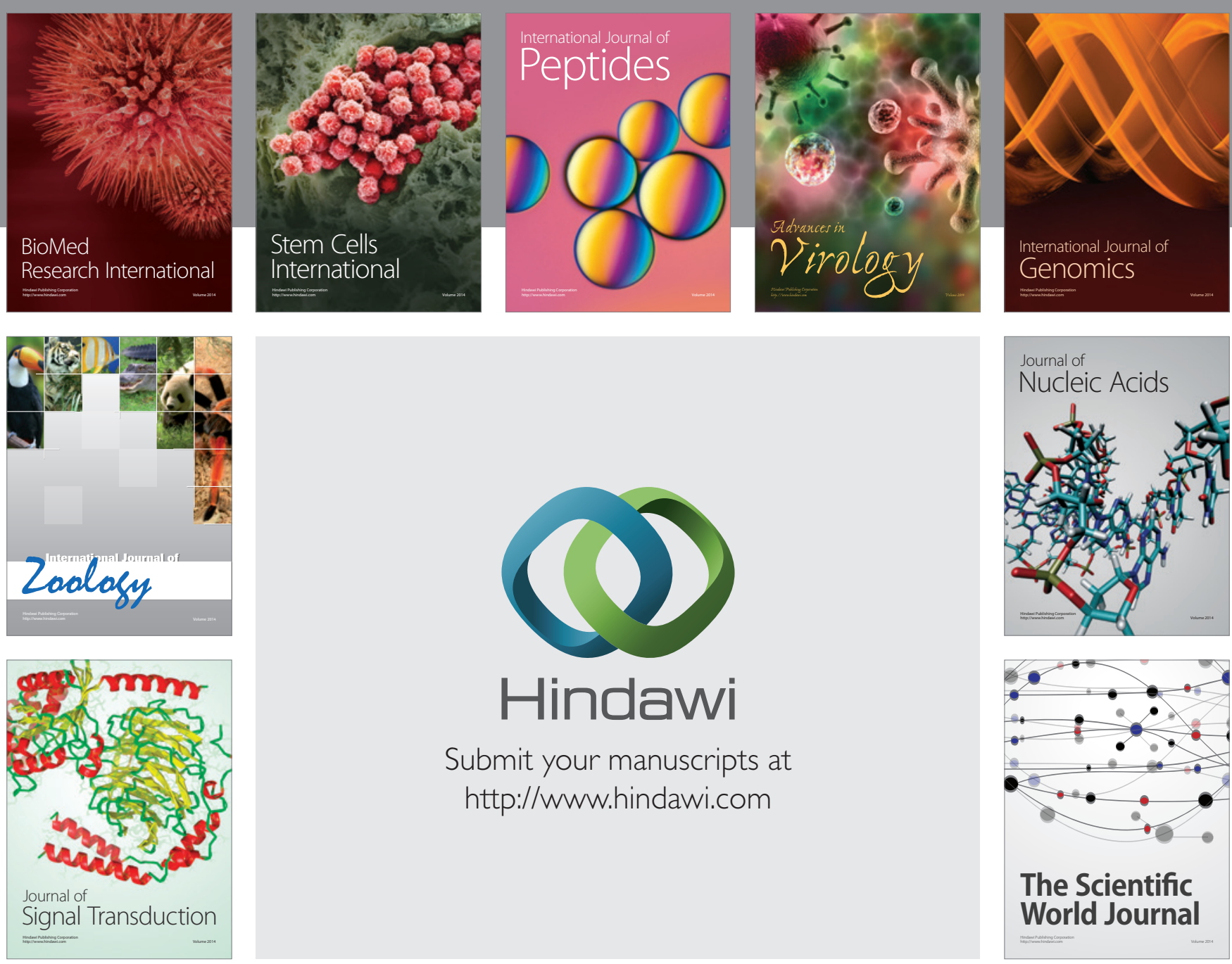

Submit your manuscripts at

http://www.hindawi.com
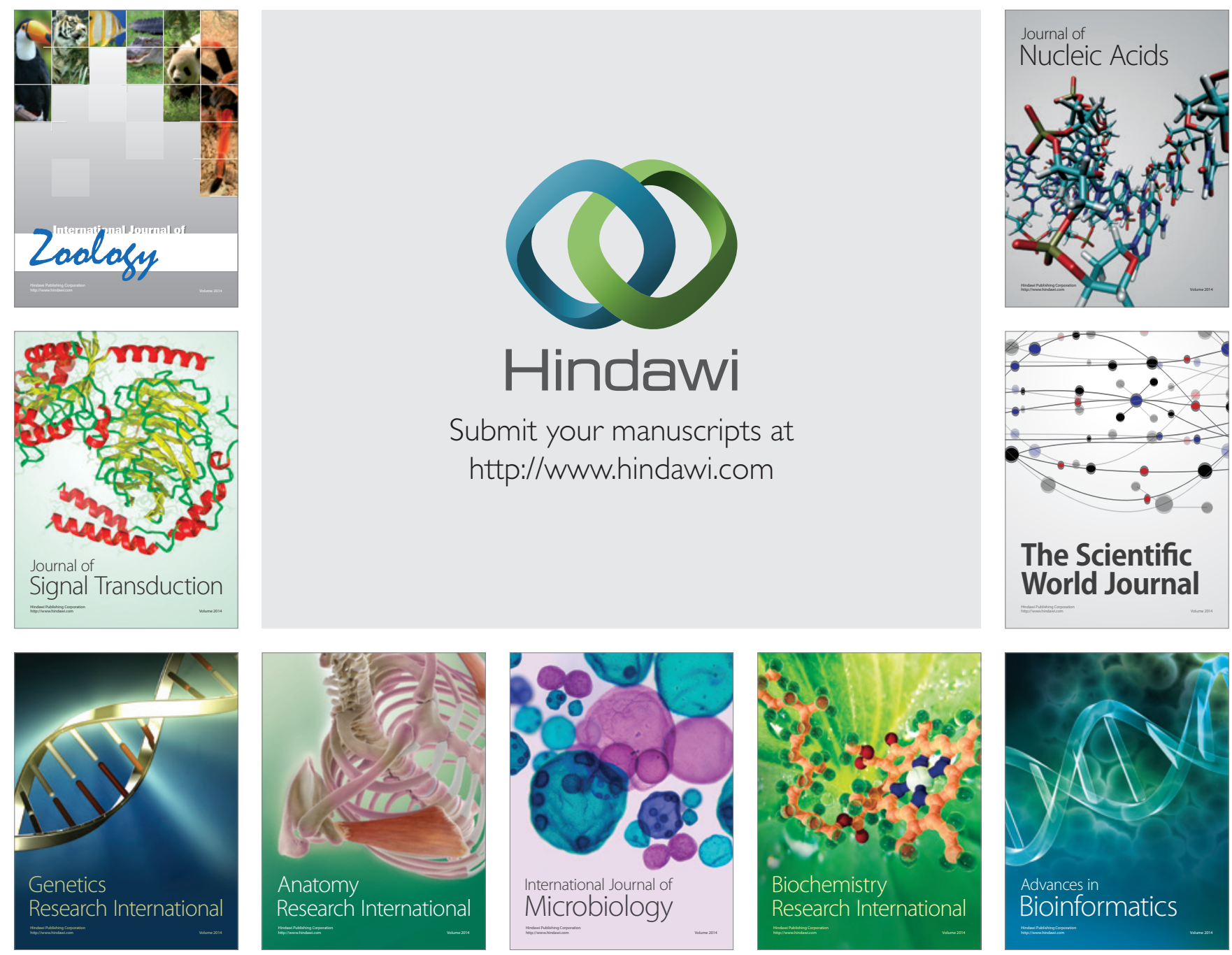

The Scientific World Journal
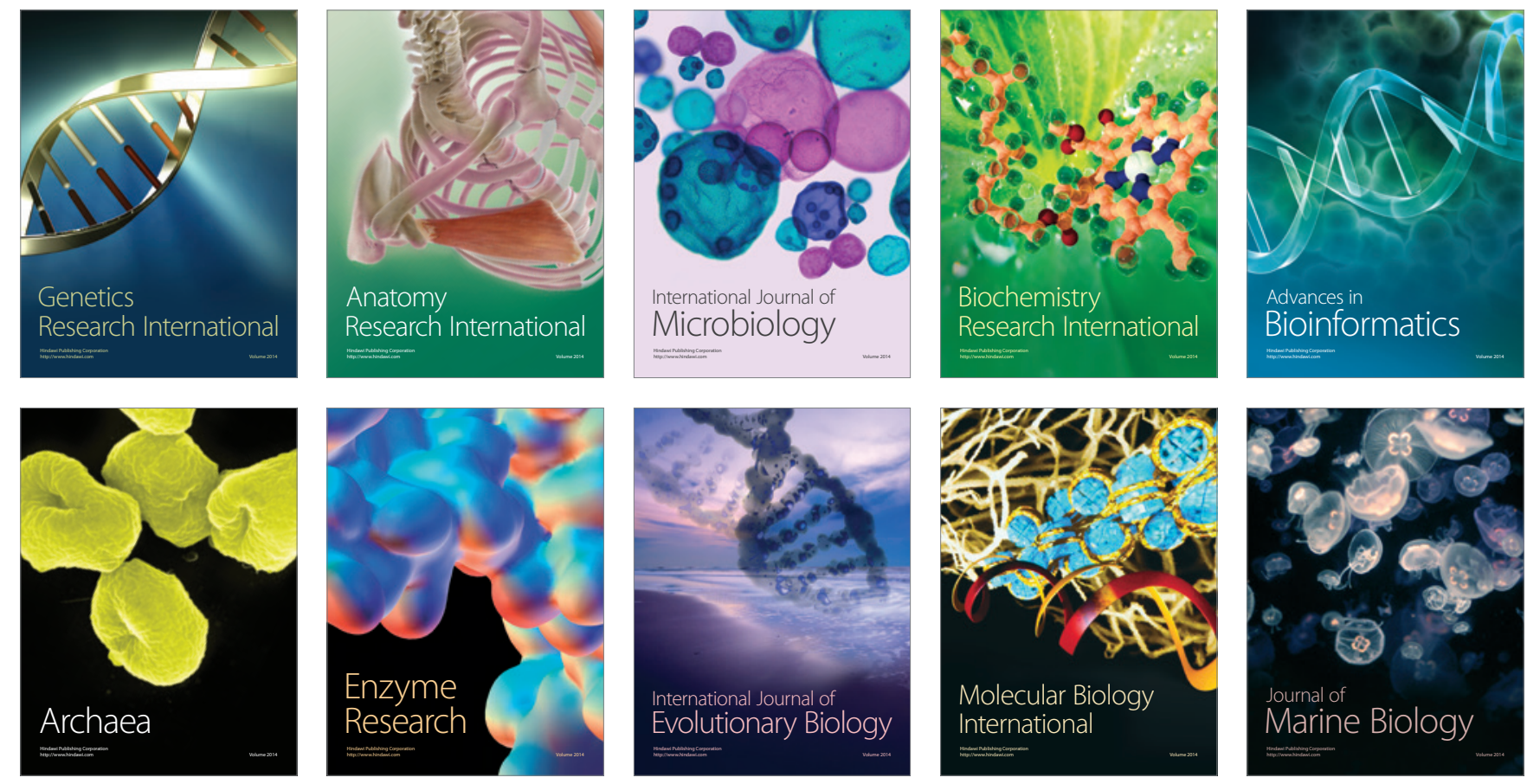